\title{
PET-MRI in the head and neck area: challenges and new directions
}

\author{
J. A. Castelijns
}

Received: 7 April 2011 / Accepted: 13 May 2011 /Published online: 23 June 2011

(C) The Author(s) 2011. This article is published with open access at Springerlink.com

\section{PET-MRI in the head and neck area: Challenges and new directions}

In the March 2011 number of European Radiology, Boss and co-authors describe their first experiences with the feasibility of simultaneous PET-MR imaging in the head and upper neck area [1]. They found that simultaneous PETMRI of head and neck cancer was feasible with a new hybrid PET-MRI prototype, using a removable brain PETdetector that can be placed within the MR gantry. They report that MRI datasets acquired during simultaneous PET data acquisition exhibited excellent image quality without any recognisable artefacts or distortions caused by the PET insert. PET datasets showed slight streak artefacts, which did not lead to degradation of tumour visualization. Images obtained with the PET-MRI system exhibited better detailed resolution and greater image contrast in comparison to those from the PET-CT system. The authors also report that full quantification of regional radiotracer activity is not yet possible. This precludes the calculation of standardized uptake values as a semi-quantitative measure of radiotracer accumulation and local uptake needs to be scaled towards a reference tissue. The authors state that this current deficit is expected to be amended soon by implementing a calibration workflow. A clear disadvantage of this PET-MRI system mainly designed for brain imaging is the small field-ofview of the PET component of approximately $19 \mathrm{~cm}$.

J. A. Castelijns $(\bowtie)$

Department of Radiology, VU University Medical Center,

De Boelelaan 1117,

1081 HV Amsterdam, The Netherlands

e-mail: j.castelijns@vumc.nl

J. A. Castelijns

PO Box 7057, 1007 MB Amsterdam, The Netherlands
Therefore the axial field of view of the PET insert typically reached to structures located cranially of the angle of the mandible, leaving the larynx and hypopharyx outside the fieldof-view. Actually the authors only report on nasopharyngeal and not even on oral and oropharyngeal carcinomas.

Magnetic resonance imaging provides unmatched soft tissue detail along with a reasonable array of functional information through techniques as MRI diffusion and MRI perfusion. However it will take a long time before MRI can provide the molecular detail that PET does and PET provides the anatomical information that MRI does, so a combination of the two technologies is the most reasonable option. Combining the two advanced imaging technologies without degrading the original optimum performance of either is challenging [2]. At present, three solutions are considered by manufacturers: a sequential, insert and integrated approach.

In the sequential construct, the PET and MRI systems are placed in a sequence. This approach is used in PET-CT and most currently available PET-MRI. However, multimodality imaging performed with separate imaging devices requires patients repositioning, which results in a greater risk of voluntary or involuntary patient movements between procedures. There are some physiologic processes such as tissue perfusion, which may change rapidly over time that consecutive data acquisition of PET and MRI may yield inaccurate results and a fully integrated system for simultaneous data acquisition is necessary. In the long term it remains to be determined if PET-MRI set-ups with sequential data acquisition capacity have a future because, especially in the mobile head and neck, there will be problems regarding reposition. In addition the options for optimizing workflow are restricted.

The insert construct system involves building a removable PET-detector that can be placed within the MR gantry. The study in the article under discussion made use of this combination: a brain PET in a slightly modified clinical 3.0 
Tesla whole body MRI. However as already stated by Boss et al. [1], such a system appears to have a restricted value for the head and neck region, because only the cranial part of the head and neck region may be imaged sufficiently.

In a third combination, a so-called integrated system simultaneous whole-body PET-MR imaging for humans is under development. The simultaneous acquisition of the PET and MRI-data allows for a better image co-registration, since motion artefacts between the 2 data acquisitions are greatly reduced. Simultaneous image acquisition enables temporal co-registration of dynamic PET data acquisition and morphological/dynamic MRI. A variety of functional information can be acquired by MRI, e.g. diffusion (proliferation, cell density, etc.) and perfusion (vessel leakage, micro-vessel density, etc.).

Simultaneous acquisition of PET and MRI may have a high value for imaging of head and neck cancer. Especially in this region, reposition may be difficult due to the high mobility of the neck and its refined anatomical structures. For assessment of clinical value, various examination protocols need to be developed in order to gain experience and explore the possibilities of the combined PET-MRI over other imaging techniques. For pre-treatment assessment of primary tumour extent and detection of regional metastases, MRI supplemented by ultrasound-guided-fine-needle-aspiration-cytology for neck diagnosis already has high value. Pre-treatment value of PET-MRI may be found by improving the detection of distant metastases. Both MRI and PET have possibilities regarding detection of distant metastases. However it may be expected that the highest value of such combined imaging for patients with head and neck cancer may be found in prediction of response after treatment with chemo-radiation, in early and late evaluation after treatment with chemo-radiation and when there is suspicion of tumour recurrence.

A meta-analysis performed by Isles et al. [3] showed that PET is highly accurate for monitoring response and detection of relapse at the primary tumour site in patients with advanced head and neck cancer after treatment with chemo-radiotherapy. The results for the neck were lower. As a result PET may have the potential to obviate the requirements for surveillance endoscopies. However it is less sensitive early after treatment and has poor anatomical detail. Obviously, a single investigation alone does not provide enough information into the relevant tumour processes. Combined information from multiple techniques such as PET-MRI can be used to monitor multiple process parameters by MRI and PET [3]. MRI and PET may be used in conjunction either to monitor the same physiological parameter for cross-validation or to monitor different stages of metabolic activity. The most precise studies to correlate and cross-validate different MR and PET perfusion measurements would be simultaneous PET-MRI. Here it is guaranteed that the tissue is at exactly the same physiological state during the experiment, since the two measurements can be performed isochronously. [4]. New horizons of multi-parametric imaging are foreseeable with PET-MRI which is not limited to a procedure that is equivalent to PET-CT, where the CT is replaced by MRI, but offers an enormous variety of research and clinical investigations in which the functional capabilities of both MRI and PET are used in a complementary way.

It is clear that head and neck imaging may gain clear advantage of PET-MRI if a system is used in which the entire head and neck area is imaged simultaneously by both techniques and not only the upper head and neck area, as in the study under discussion. Therefore we have to wait for an integrated system in which simultaneous whole-body PETMRI is possible. Problems like slight streak artifacts in PET datasets and regarding full quantification of regional radiotracer activity should be solved. Algorithms and procedures have been developed for MR based PET attenuation correction [5]. For a long time, this hardware challenge limited the ability of PET-MRI to compete with PET-CT or PET in terms of quantification. Because these hurdles seem to be successfully overcome, the first whole-body PET-MRI systems are expected soon in clinical practice. It may then be expected that integrated PET-MRI for head and neck imaging is likely to be formidable research tool and, in the near future, a routine clinical investigation.

Open Access This article is distributed under the terms of the Creative Commons Attribution Noncommercial License which permits any noncommercial use, distribution, and reproduction in any medium, provided the original author(s) and source are credited.

\section{References}

1. Boss A, Stegger L, Bisdas S, Kolb A, Schwenzer N, Pfister M, Claussen CD, Pichler BJ, Pfannenberg C (2011) Feasibility of simultaneous PET/MR imaging in the upper head and neck area. Eur Radiol 21:1439-46

2. Daftary A (2010) PET-MRI: challenges and new directions. Indian J Nucl Med 25:3-5

3. Isles MG, McConkey C, Mehanna HM (2008) A systematic review and meta-analysis of the role of PET in the follow-up radiotherapy or chemotherapy. Clin Otolaryngol 33:210-222

4. Wehrl HF, Sauter AW, Judenhofer MS, Pichler BJ (2010) Combined PET-MR imaging-technology and applications. Trends Mol Med 16:508-15

5. Hofmann M, Steinke F, Scheel V et al (2008) MR-based attenuation correction for PET/MRI: a novel approach combining pattern recognition and atlas registration. J Nucl Med 49:1875-1883 